\title{
O Tratamento Antiplaquetário por Via Oral na Doença Arterial Coronária - Visão Atual
}

\author{
Current Antiplatelet Oral Treatment for Coronary Artery Disease: \\ A Present View
}

Gilson Soares Feitosa ${ }^{1}$, Gilson Soares Feitosa-Filho ${ }^{1}$, Paulo Barbosa ${ }^{1}$, Rosenbert da Silva ${ }^{1}$, Thiago Pereira ${ }^{1}$, Isabela Pilar ${ }^{1}$, Marcus Vinicius Andrade ${ }^{1}$, Antonio Carlos Sales Nery ${ }^{1}$

${ }^{1}$ Serviço de Cardiologia do Hospital Santa Izabel; Salvador, Bahia, Brasil

Correspondence addresses: Dr. Gilson Soares Feitosa gilson-feitosa@uol.com.br

Received: August 23, 2019

Revised: August 30, 2019

Accepted: September 11, 2019

Published: December 2 $2^{\text {nd }}, 2019$

Data Availability Statement: All relevant data are within the paper and its Supporting Information files.

Funding: This work was the result of authors' initiative. There was no support of research or publication funds.

Competing interests: The authors have declared that no competing interests exist.

Copyright

(C) 2019 by Santa Casa de Misericórdia da Bahia.

All rights reserved.

ISSN: 2526-5563
$O$ reconhecimento da importância que desempenha a trombose intracoronária no desenvolvimento do processo aterosclerótico e seus desfechos clínicos fez com que esforços se voltassem para a compreensão do papel das plaquetas nesse processo e, consequentemente, da maneira de evitar sua propriedade de agregação que culmina com a progressiva formação do trombo oclusor da luz do vaso com suas consequências isquêmicas para o tecido miocárdico (angina, infarto, morte súbita e insuficiência cardíaca). Após o estabelecimento do ácido acetilsalicílico (aspirina) e sua importante ação antiagregante plaquetária, com comprovados benefícios clínicos, o desenvolvimento na área se faz à procura de novos agentes e sua oportunidade de uso diante das numerosas condições que se oferecem para o seu emprego. Dessa forma, surgem para uso por via oral diversos fármacos com ação em sítios distintos daquele da ação da aspirina na superfície da plaqueta, destacando-se, sobremaneira, aqueles que inibem o receptor $\mathrm{P2Y12}$, que reforçam a ação antiplaquetária da aspirina quando supra juntados a esta ou mesmo em substituição à mesma. Por outro lado, o desvendamento e caracterização progressivos dos quadros de apresentação da doença coronariana, com sua multiplicidade de tratamentos, fornecem cenários os mais variados, a exigir desses fármacos uso mais proveitoso. Isto porque, juntamente com o resultado da ação principal, antitrombótica, que previne eventos isquêmicos, associa-se, necessariamente, um risco aumentado de sangramento, que deve ser considerado quando do emprego destes importantes agentes no tratamento da doença coronariana estabelecida. $O$ objetivo dessa atualização é o de rever à luz de trabalhos de alta qualidade metodológica geralmente comparativos, randomizados e, na maioria das vezes, duplos-cego - as melhores evidências que justificam a escolha do agente antiplaquetário, ou sua combinação, pelo tempo de uso considerado adequado para as diversas situações em doença coronariana em que surja a oportunidade do seu uso.

Palavras-chave: Antiplaquetário; Doença Coronariana Estável; Síndrome Coronariana Aguda; Infarto do Miocárdio; Fibrilação Atrial.

The progressive recognition of the importance played by intracoronary thrombosis in the development of the atherosclerotic process and the clinical outcomes led to a better understanding of the role of the platelets in this process and how to find a way to avoid their aggregation, which leads to the progressive thrombus growth, vessel occlusion and undesirable ischemic effects to the myocardial tissue cells (angina, infarct, sudden death, and heart failure). Since the discovery of the salicylic-acetic acid (aspirin) and its antiplatelet property with clinical benefits, further developments 
in the area pursued the discovery of new agents and distinct uses in the face of the different conditions that arise for the consideration of their utilization. Thus, several drugs appearing for oral use at different sites from the action of aspirin on the platelet surface, especially those that inhibit the P2Y12 receptor, which reinforce the antiplatelet action of aspirin when added to that or even in place of it. On the other hand, the progressive recognition of the several clinical conditions of the coronary artery disease, with a lot of treatment forms, presents many distinct scenarios that demand the best choice of antiplatelet use. This is because, together with the outcome of the main antithrombotic action that prevents ischemic events, there is necessarily associated an increased risk of bleeding, which should be considered when employing these important agents in the treatment of established coronary disease. The purpose of this update is to review articles with high quality of methods purposes - usually comparative, randomized, and most often double-blind - the best evidence to justify the choice of antiplatelet agent, or combination thereof, by time. considered appropriate for the various situations in coronary artery disease in which the opportunity for its use arises. This review aims to define the optimal choice of the antiplatelet agent, or its combination, for the appropriate length of time, in the several situations of coronary artery disease, considering a detailed analysis of the best methodologically carried out papers, usually comparative, prospective, randomized and double-blind studies. Keywords: Antiplatelet Agents; Stable Coronary Artery Disease; Acute Coronary Syndrome; Acute Myocardial Infarction; Atrial Fibrillation.

\section{Introdução}

Reconhecidos progressos foram desenvolvidos no tratamento clínico da doença arterial coronária, principalmente, com ações higienodietética e farmacológicas que atuam estabilizando a placa aterosclerótica no interior das coronárias.

Dentre os recursos farmacológicos, destacamse, sobremaneira, os antiplaquetáriose as estatinas, além dos medicamentos que atuam no sistema renina-angiotensina e os betabloqueadores.

Estudos recentes comparativos de tratamento clínico versus invasivo, como intervenção percutânea coronária ou cirurgia de revascularização, têm servido, de maneira convincente, para realçar a importância do tratamento clínico moderno, na garantia da manutenção dos resultados a longo prazo. ${ }^{1}$

Ademais, os estudos COURAGE ${ }^{2}$ e o ISCHEMIA, este último recentemente apresentado no AHA19, entre os mais contemporâneos, demonstraram resultados comparáveis em uma abordagem inicial de pacientes com doença coronariana estável, entre tratamento clínico e de revascularização, em termos de eventos coronarianos sólidos, em períodos de acompanhamento de 3 a 7 anos.

A base fundamental comprovada de benefícios com tratamento farmacológico é sua ação direta na aterotrombose.Tal tem sido comprovado com os antiplaquetários, as estatinas e os inibidores do sistema reninaangiotensina.

O fenômeno trombótico desempenha um importante papel na oclusão do vaso coronariano assim como na progressão da placa. Eventual rotura da placa coronária ou erosão endotelial expõe o colágeno da parede arterial ao fluxo sanguíneo, proporcionando o desencadeamento de formação do trombo, elemento principal na oclusão do vaso, determinando a síndrome coronariana aguda. Além disso, eventualmente, o trombo leva à progressão do tamanho da placa existente por estimular ambiente adicional próinflamatório, com consequente acúmulo de LDL e migração de células inflamatórias para o interior da placa, ocasionando o seu crescimento na íntima dos vasos. Os antiplaquetários cumprem principalmente o propósito de diminuir a formação do trombo em caso de erosão ou rotura da placa e também servem para estabilizar o crescimento da mesma.

\section{Aspirina}

$\mathrm{O}$ acido acetilsalicílico tem sido usado para essa finalidade há muitos anos e embora tenha o potencial de causar gastrite e hemorragias no estômago e em outras áreas, tem um balanço positivoentreaprevenção do fenômeno isquêmico e a ocorrência de hemorragias, sendo, portanto, 
um antiplaquetário muito utilizado há muito. Sua ação antiplaquetária deve-se à acetilação irreversível da cicloxigenase, ${ }^{3}$ impedindo a formação de tromboxane A2 a partir do ácido araquidônico e, assim, ao final, reduz a exposição e mudança conformacional da glicoproteína IIbIIIa, que é o elemento indispensável para a agregação plaquetária.

\section{Outros Antiplaquetários}

Vários outros elementos da superfície plaquetária são reconhecidos como possíveis deflagradores de mudança da plaqueta que culminam na mesma expressão da glicoproteína IIbIIIa, respondendo a vários estímulos, e que têm sido alvo de interesse para sua inibição (Figura 1).

Os mais bem-sucedidos têm sido os receptores de adenosina P12Y2, dando origem a um grupo específico de antiplaquetários, como a ticlopidina, o clopidogrel, o ticagrelor, o prasugrel, entre outros.

A ticlopidina, embora tivesse a mesma eficácia que o clopidogrel em prevenir eventos isquêmicos, como demostrado no estudo comparativo de ticlopidina versus clopidogrel em 1.020 pacientes testados no ensaio CLASSICS, ${ }^{4}$ por conta de seus possíveis efeitos colaterais hematológicos de leucopenia, tem sido menos utilizada.

Os novos antiplaquetários de uso mais recente, ticagrelor e prasugrel, apresentam-se como

Figura 1. A plaqueta com seus receptores.

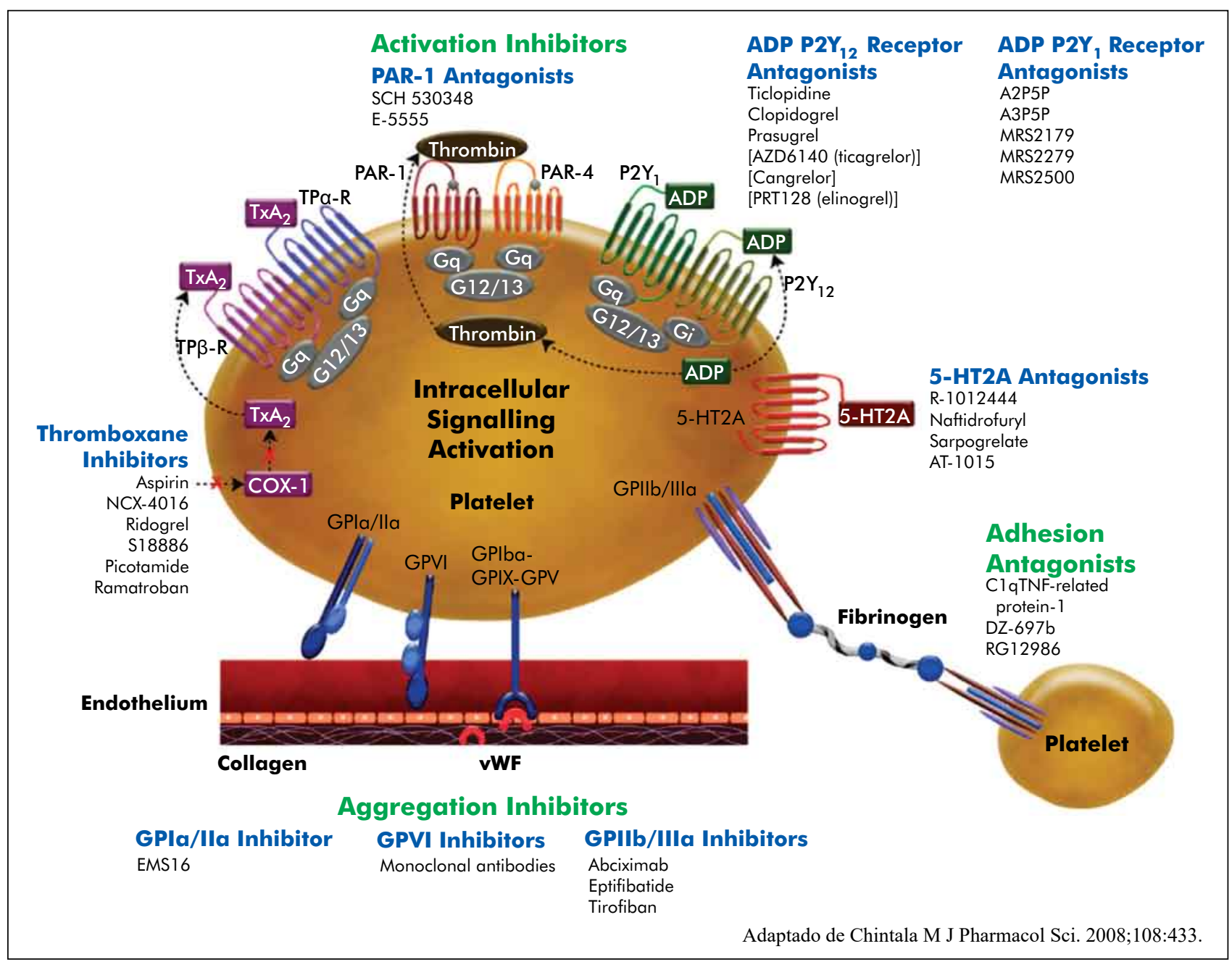


inibidores do P2Y12, ambos com maior ação de antiagregação plaquetária que o clopidogrel, além de início de ação mais rápida.

O Quadro 1 apresenta a diversidade muito grande de situações que envolvem a doença coronariana em suas manifestações e decisões terapêuticas e o emprego dos antiplaquetários.

Quadro 1. Cenários de utilização do tratamento antiplaqueário em doença coronariana.

1. Antiplaquetário em DAC estável

1.1. Monoterapia em DAC estável.

1.2. Terapêutica antiplaquetária dupla em DAC estável.

1.3. Terapia antiplaquetária e anticoagulante no paciente com DAC estável.

2. Terapia antiplaquetária em síndrome coronariana aguda.

2.1. Monoterapia antiplaquetária em síndrome coronariana aguda.

2.2. Terapêutica antiplaquetária dupla em síndrome coronariana aguda.

2.3. Os novos antiplaquetários em síndrome coronariana aguda.

2.4. Anticoagulantes e antiplaquetários em síndrome coronariana aguda sem fibrilaçao atrial.

2.5. Duração da terapêutica antiplaquetária dupla após o uso de stents.

2.5.1. 1 mês

2.5.2. 3 meses

2.5.3. 1 ano ou mais

2.6. Antiplaquetários após uso de stents em procedimentos eletivos.

2.7. O uso de antiplaquetários no portador de DAC com fibrilação atrial.

2.8. Terapêutica antitrombótica em FA e DAC estável.

3. Interrupção da terapêutica antiplaquetária.

\section{Antiplaquetários em DAC Estável}

\subsection{Monoterapia em DAC Estável}

O emprego da aspirina em DAC estável está bem estabelecido.

Em 287 estudos compreendendo um total de 13.5000 pacientes portadores de doença cardiovascular ou risco de Framingham acima de $10 \%$, a aspirina foi testada contra um grupo controle para prevenção de eventos vasculares maiores: morte vascular, infarto não-fatal $\mathrm{e}$ acidente vascular não-fatal. Houve uma redução sistemática de cerca de $25 \%$ de desfechos. ${ }^{5}$

A dose recomendada, tendo em vista o equilíbrio entre a sua eficácia anti-isquêmica e risco de hemorragia, tem sido de $100 \mathrm{mg}$ ao dia.

O estudo ADAPTABLE (NCT02697916), ainda em andamento, testa se a dose deveria ser maior em pacientes com peso acima de $70 \mathrm{~kg}$.

O uso de antiplaquetário deverá ser permanente à luz dos conhecimentos atuais, geralmente como monoterapia e, na maioria dos casos, com o emprego da aspirina.

Outras formas de monoterapia antiplaquetária emDAC estável já foram testadas, principalmente com o clopidogrel.

O principal estudo comparativo entre as duas estratégias de tratamento foi o CAPRIE, ${ }^{6} \mathrm{com}$ 19.185 pacientes, que comparou o uso de aspirina $325 \mathrm{mg} /$ dia versus o uso de clopidogrel $75 \mathrm{mg} /$ dia - um estudo duplo-cego, randomizado, em portadores de doença coronariana com infarto ocorrido há menos de 35 dias, acidente vascular cerebral há mais de 1 semana e menos que 6 meses, ou doença arterial periférica.

$\mathrm{O}$ desfecho primário combinado (infarto do miocárdio, ou acidente vascular ou morte cardiovascular) em 36 meses ocorreu em $5,32 \%$ e $5,83 \%$ para clopidogrel e aspirina, respectivamente, com redução de risco de $8,7 \%$ para o grupo clopidogrel $(p=0,043)$. Não houve diferença na mortalidade total. Sangramento de qualquer tipo ocorreu de forma igual nos dois grupos em 9,27 e 9,28\% para clopidogrel e aspirina, respectivamente, e dentre estes apenas 
o sangramento digestivo foi mais frequente com a aspirina $(2,66 \%)$ do que com o clopidogrel $(1,99 \%)(\mathrm{p}<0,05)$.

Em metanálise de estudos comparativos de clopidogrel versus aspirina confirmam-se estes achados.

A rigor, não existem estudos comparativos diretos de aspirina, ou do clopidogrel, em doença arterial coronariana estável, com os novos antiagregantes ticagrelor ou prasugrel, exceto no contexto de síndrome coronariana aguda ou com implante de stents ou mesmo com uso de anticoagulantes simultaneamente para tratamento de FA.

\subsection{Terapêutica Antiplaquetária Dupla em DAC Estável}

Considerando-se os distintos mecanismos de ação das drogas, tem-se investigado sobre o emprego da dupla antiagregação plaquetária em doença coronariana estável. Por agirem em receptores distintos da superfície plaquetária, a combinação de aspirina com um inibidor da receptor PDY12 tem merecido atenção.

$\mathrm{O}$ estudo $\mathrm{CHARISMA}^{7}$ testou essa hipótese em 15.603 portadores de doença cardiovascular ou de múltiplos fatores de risco para doença coronariana, comparando AAS + clopidogrel com AAS e placebo, tendo como desfecho primário a combinação de infarto do miocárdio, acidente vascular cerebral ou morte de origem cardiovascular. $\mathrm{O}$ desfecho primário ocorreu, em uma média de 28 meses de acompanhamento, em $6,8 \%$ e $7,3 \%$ para o grupo do clopidogrel e o grupo placebo, respectivamente, com risco relativo de 0,93 ( $\mathrm{p}=0,22$, não significativo).

$\mathrm{O}$ risco de sangramento grave foi $1,7 \%$ e $1,3 \%$ para os grupos clopidogrel e controle, respectivamente, com risco relativo de $1,25 \%$ $(p=0,09)$, mostrando tendência a maior sangramento no grupo clopidogrel.

Naqueles pacientes com doença estabelecida, o desfecho primário ocorreu em 6,9\% e 7,9\% para o grupo clopidogrel e placebo, respectivamente, com risco relativo de $0,88(\mathrm{p}=0,046)$, sugerindo um benefício, embora com uma margem pequena.

No estudo PEGASUS, ${ }^{8}$ essa hipótese de dupla terapêutica antiplaquetária foi testada com o emprego de ticagrelor + aspirina comparada a aspirina e placebo em 21.162 pacientes portadores de DAC e que tivessem apresentado um infarto em período superior a 1 anos antes do ingresso no estudo. O estudo teve uma duração média de 33 meses. O desfecho primário, constituído por uma combinação de morte cardiovascular, acidente vascular cerebral ou infarto, foi visto em 7,85\% no grupo ticagrelor $90 \mathrm{mg} 2$ vezes ao dia; 7,77\% no grupo ticagrelor $60 \mathrm{mg} 2$ vezes ao dia, quando comparados a 9,04\% no grupo placebo com risco relativo, apresentando redução de 15 e 16\%, respectivamente, em relação ao placebo $(p=0,008$ e 0,004 , altamente significativo). Houve, porém, incremento apreciável de sangramento maior de $6 \%$ em ambos os grupos $(\mathrm{p}<0,001)$. Convém notar que dentre esses sangramentos, a hemorragia cerebral foi igual nos 3 grupos (menos de 1\%).

Em subsequente análise do estudo PEGASUS, limitado aos casos de portadores de doença multiarterial, que constituiu um total de 12.558 pacientes, os resultados do beneficio foram mais expressivos, da ordem de $18 \%$, e, embora houvesse um aumento de sangramento em geral, não houve incremento de sangramento intracerebral ou hemorragia fatal.

\section{$\underline{1.3 \text { Terapia Antiplaquetária e Anticoagulante no }}$} $\underline{\text { Paciente com DAC Estável }}$

A ideia de uma terapêutica antitrombótica mais potente utilizando simultaneamente um antiplaquetário e um anticoagulante em DAC estável parece atraente tendo-se em conta sua ação sinérgica antitrombótica agindo contra a agregação plaquetária e a formação de fibrina. Tais vantagens anti-isquêmicas, se comprovadas, teriam que ser avaliadas contra as desvantagens de um esperado aumento nas chances de sangramento. Dadas as vantagens 
comprovadas dos novos anticoagulantes sobre aqueles antivitamina $\mathrm{K}$, o maior interesse recaiu em testar os novos anticoagulantes juntamente com antiplaquetários nesta condição. Ainda não está disponível, todavia, estudos dessa natureza fora do contexto de fibrilação atrial.

\section{Terapia Antiplaquetária em Síndrome Coronariana Aguda}

2.1. Monoterapia Antiplaquetária em Síndrome Coronariana Aguda

Em infarto do miocárdio com supra desnivelamento do segmento ST, a aspirina teve seu papel definitivamente assentado quando foi comparada ao uso do fibrinolítico estreptoquinase no estudo basilar ISIS-2. ${ }^{9}$ Neste estudo, 17.187 pacientes com infarto com supra de ST foram comparados: o uso oral de aspirina $160 \mathrm{mg}$ versus estreptoquinase a placebo, demonstrando equivalência entre as duas substâncias em prevenir desfechos clínicos, como mortalidade vascular, ambos isoladamente melhores que placebo. Ademais, os dois usados em conjunto tiveram os melhores resultados para mortalidade vascular: 9,4\%, 9,2\%, $12 \%$ e $8 \%$, respectivamente, em 35 dias. Resultado que se manteve em acompanhamento médio por 15 meses.

\subsection{Terapêutica Antiplaquetária Dupla em} Síndrome Coronariana Aguda

A terapêutica antiplaquetária dupla, reunindo a aspirina e o clopidogrel, tem se confirmado como de grande utilidade em síndrome coronariana aguda quando usada em um seguimento por 1 ano. O estudo $\mathrm{CURE}^{10}$ testou 12.562 pacientes com síndrome coronariana aguda (excluindo-se os casos de IAM com supra de ST) com adição de clopidogrel $300 \mathrm{mg}$ bolus nas primeiras 24 horas dos sintomas e depois $75 \mathrm{mg}$ de aspirina na dose variável de 75 a $325 \mathrm{mg}$, por um período de 3 meses a 1 ano, em comparação ao uso isolado de aspirina. Os resultados demonstraram uma redução dos desfechos combinados de morte cardiovascular, IAM não fatal e acidente vascular cerebral de $9,3 \%$ e $11,4 \%$, respectivamente, para clopidogrel versus placebo, com redução de risco relativo de $20 \%(p=0,0005)$. Houve ligeiro aumento de sangramento.

Os benefícios isquêmicos foram realmente registrados nos primeiros 3 meses do acompanhamento, persistindo-se por 1 ano. A despeito desses achados, justificam-se as recomendações até hoje do emprego da terapêutica antiplaquetária dupla em seguida a uma síndrome coronariana aguda por 1 ano, até naqueles que seguiram para realização de um procedimento de revascularização cirúrgica.

Em IAM com supra de ST, este benefício também foi registrado com base no estudo chinês COMMIT-CCS, ${ }^{11}$ que envolveu 45.852 pacientes, $93 \%$ com IAM com supra de ST ou bloqueio completo do ramo esquerdo. $\mathrm{O}$ estudo igualmente demonstrou o benefício do uso de clopidogrel adicionado à aspirina no tratamento de pacientes com essa síndrome coronariana aguda por 28 dias.

O estudo CLARITY ${ }^{12}$ também confirma esses resultados em 30 dias com redução de eventos similares em $20 \%$.

A tentativa de usar dose de impregnação de clopidogrel de $600 \mathrm{mg}$ e manutenção de $150 \mathrm{mg}$ por 7 dias para uso posterior de $75 \mathrm{mg} /$ dia versus impregnação de $300 \mathrm{mg}$ e posterior uso de $75 \mathrm{mg}$, foi testada em 25.086 pacientes com síndrome coronariana aguda no estudo CURRENT OASIS $7 . .^{13}$ Os resultados demostraram-se benéficos em pacientes que se submeteram a intervenção coronariana precoce com redução de eventos isquêmicos de $14 \%$ e de trombose do stent em $46 \%$, com pequeno incremento no risco de sangramento. Nos pacientes que não se submeteram a intervenção coronariana precoce não houve benefício e houve aumento de hemorragias.

\subsection{Os Novos Antiplaquetários em Síndrome} Coronariana Aguda

O surgimento dos novos antiplaquetários, 
inibidores do receptor P2Y12, aponta para maior eficácia anti-isquêmica destes antiplaquetários mais recentes, sem prejuízo maior relacionado a hemorragias.

Os novos antiplaquetários disponíveis, ticagrelor e prasugrel, apresentam a farmacologia de ação melhor e de maior intensidade de antiagregação que o clopidogrel.

No estudo PLATO (1 ano de acompanhamento), ${ }^{14}$ com 18.624 pacientes portadores de síndrome coronariana aguda, com e sem supra de ST, com acréscimo da aspirina, o ticagrelor em dose de impregnação de $180 \mathrm{mg}$ seguida de dose de manutenção de $90 \mathrm{mg} 2$ vezes ao dia em comparação ao clopidogrel 300 a 600 $\mathrm{mg}$, seguido de $75 \mathrm{mg}$ ao dia, ofereceu melhores resultados na redução de desfechos combinados de morte cardiovascular, infarto do miocárdio e acidente vascular cerebral de $16 \%(\mathrm{p}<0,001)$.

De forma individual, esses benefícios foram vistos na mortalidade cardiovascular e infarto do miocárdio, mas não em acidente vascular cerebral.

A mortalidade total ocorreu em 4,5\% e 5,9\% para ticagrelor e clopidogrel, respectivamente $(p=0,03)$.

A ocorrência de sangramento foi de $11,6 \%$ e $11,2 \%$ para ticagrelor e clopidogrel, respectivamente, sem diferença significativa, que somente foi registrada com predomínio do ticagrelor naqueles pacientes que se submeteram à cirurgia de revascularização.

Convém salientar que nesse estudo o antiplaquetário foi ministrado na sala de emergência (upfront).

No subgrupo de síndrome coronariana aguda, que teve a intervenção percutânea planejada antes da randomização, houve uma diminuição do desfecho primário a favor do ticagrelor de $10,7 \%$ e $9 \%$, respectivamente, com redução do risco relativo de $16 \%(\mathrm{p}<0,001)$. Os pacientes que permaneceram em tratamento clínico obtiveram uma redução de $14,3 \%$ para $12 \%$ a favor do ticagrelor, com redução do risco relativo de $15 \%$ $(p=0,045)$.
A queixa mais frequente foi de dispneia, principalmentenos primeiros meses de tratamento no grupo do ticagrelor em comparação ao grupo do clopidogrel, de $13,8 \%$ e 7,8\% (p<0,001). Essa dispneia levou à suspensão da droga em apenas $0,9 \%$ dos casos com ticagrelor e $0,1 \%$ no grupo clopidogrel $(p<0,001)$. Investigação subsequente revelou que a causa da dispneia se deve ao transitório acúmulo de ADP e não por razões de qualquer dano tecidual.

O prasugrel foi testado em comparação ao clopidogrel com 13.608 pacientes no contexto da síndrome coronariana aguda, incluindo todo o seu espectro de angina instável, infarto com e sem supra de ST, com planejamento de realização de intervenção percutânea no estudo TRITON. ${ }^{15}$ Em todos os casos para uso de clopidogrel $300 \mathrm{mg}$ (dose de impregnação) seguido de $75 \mathrm{mg} 1 \mathrm{vez}$ ao dia, ou prasugrel $60 \mathrm{mg}$ de impregnação seguido de $10 \mathrm{mg}$ por dia, a randomização foi feita após conhecimento da anatomia coronária (upstream) e decisão de proceder com a angioplastia, exceto em 2.438 pacientes com IAM com supra de ST, em um contexto de angioplastia primária, se o cateterismo foi realizada nas primeiras 12 horas desde o início dos sintomas, quando então a randomização foi feita de imediato.

Os resultados mostraram redução de desfecho primário de morte cardiovascular, IAM não fatal ou AVC não fatal em 19\% $(p=0,001)$. Esses benefícios foram registrados em todos os tipos de síndrome coronariana.

Ao cabo de 15 meses, a incidência de sangramento maior ocorreu em $2,4 \%$ dos pacientes tratados com prasugrel e $1,8 \%$, com clopidogrel, com aumento de risco relativo de $32 \%(p=0,03)$, principalmente em idosos $>75$ anos, de baixo peso $<60 \mathrm{~kg}$ ou com história de AVC prévio.

Dois questionamentos surgiram em relação ao estudo TRITON:

A.Momento de Emprego da Dose de
Impregnação
Diferentemente do estudo PLATO, em 
que houve uso na chegada de impregnação com ticagrelor, a maioria dos pacientes do estudo TRITON receberam a dose de impregnação do prasugrel após a realização da cinecoronariografia. Por isso, de modo a verificar a diferença em eficácia e sangramento nas duas estratégias - upfront e upstream - realizou-se o estudo ACCOAST, ${ }^{16}$ em que 4.033 pacientes com SCA sem supra de ST foram randomizados para o uso de prasugrel na chegada, na dose de $30 \mathrm{mg}$, e, uma vez submetidos a coronariografia que recomendava a angioplastia, recebiam então a dose complementar de mais $30 \mathrm{mg}$, ou para o grupo controle que recebeu $60 \mathrm{mg}$ durante a angioplastia.

Os resultados foram semelhantes no que concerne aos efeitos anti-isquêmicos, porém houve um incremento significativo de sangramento nos que utilizaram já na chegada. Dessa forma, não houve reconhecida vantagem com o emprego do prasugrel antes da realização do cateterismo.

\section{B. Há Superioridade do Prasugrel ao Clopidogrel} em Pacientes com Síndrome Coronariana Aguda sem Revascularização?

No estudo TRITON, a quase totalidade dos pacientes se submeteu à intervenção percutânea com implante de stents. No intuito de saber se havia superioridade do prasugrel ao clopidogrel, realizou-se o estudo randomizado TRILOGY$\mathrm{ACS},{ }^{17}$ com 7.243 pacientes que não tinham intenção de tratamento com revascularização nos próximos 10 dias desde o evento agudo, com um acompanhamento médio de 17 meses

Naqueles com idade menor que 75 anos, foram randomizados para $30 \mathrm{mg}$ de prasugrel e manutenção de $10 \mathrm{mg}$ diários, ou, clopidogrel $300 \mathrm{mg}$ seguido de $75 \mathrm{mg}$ diários. Naqueles com idade maior que 75 anos ou peso menor que 60 $\mathrm{kg}$, a manutenção de prasugrel foi com $5 \mathrm{mg}$.

Os desfechos combinados de morte cardiovascular, infarto do miocárdio ou acidente vascular cerebral foram semelhantes nos grupos prasugrel e clopidogrel em acompanhamento por 30 meses, embora as curvas passassem a divergir a favor do prasugrel nos últimos meses de observação. A incidência de sangramento foi semelhante em ambos os grupos.

Portanto, nos pacientes com síndrome coronariana aguda que permaneceram em tratamento clínico, prasugrel não foi superior ao clopidogrel.

Um estudo comparativo de ticagrelor e prasugrel foi recentemente publicado (ISARREACT $5,{ }^{18}$ financiado pelo German Center for Cardiovascular Research and Deutsches Herzzentrum München). Este estudo aberto, randomizado, em pacientes com síndrome coronariana aguda, no seu amplo espectro de angina instável, infarto com supra ou infarto sem supra de ST, tinha como meta realização de intervenção percutânea.

O uso do ticagrelor ocoreu na entrada (upfront) e o do prasugrel depois da realização da cineangiocoronariografia (exceto nos casos de infarto com supra de ST, quando foi administrado imediatamente), à semelhança dos estudos originais comparativos ao clopidogrel.

O ticagrelor foi usado em uma dose de 180 $\mathrm{mg}$ de ataque e depois uma manutenção de 90 mg 2 vezes ao dia. O prasugrel foi usado com uma dose de ataque de $60 \mathrm{mg}$ e manutenção de $10 \mathrm{mg}$ ao dia, exceto para pacientes com mais de 75 anos de idade ou aqueles com peso aquém de $60 \mathrm{~kg}$, que utilizaram a manutenção de $5 \mathrm{mg}$ ao dia.

O desfecho primário deste estudo foi morte, infarto ou AVC em 1 ano.

Em um total de 4.018 pacientes randomizados, a maioria procedeu com intervenção percutânea e um pequeno número fez tratamento clínico ou cirurgia de revascularização.

Houve uma vantagem de $36 \%$ do prasugrel em relação ao ticagrelor na redução de desfechos primários $(\mathrm{p}=0,006)$.

Uma análise individualizada dos componentes do desfechoprimário verifica-se que a mortalidade e AVC foram semelhantes entre os grupos e a diferença se deveu a uma menor ocorrência 
de infarto no grupo prasugrel, $4,8 \%$ e $3,0 \%$, respectivamente, para ticagrelor e prasugrel, com uma redução de risco relativo de $37 \%$.

Vale lembrar que a evidência favorável ao emprego do ticagrelor e prasugrel sobre o clopidogrel ocorreu em um contexto de síndrome coronariana aguda e emprego de stent nessa condição. O emprego dos novos antiplaquetários em intervenção percutânea eletiva tem sido feita por extrapolação. Há apenas um estudo recentemente publicado nesta condição, o STEEL-PCI, que testou o efeito antiplaquetário de ticagrelor em 162 pacientes com doença coronariana estável submetidos a angioplastia eletiva em dois regimes de ticagrelor, com $60 \mathrm{e}$ $90 \mathrm{mg} 2$ vezes ao dia, após a dose de ataque de $180 \mathrm{mg}$, contra clopidogrel $600 \mathrm{mg}$, se menos de 4 horas antes do procedimento, ou $75 \mathrm{mg}$ por 5 dias antes do mesmo, seguidos em ambos os casos de $75 \mathrm{mg}$ diários. $\mathrm{O}$ estudo se prestou à avaliação de intensidade de ação antiplaquetária que foi maior nos dois regimes de ticagrelor do que no de clopidogrel. Entretanto, não teve poder para avaliar desfechos clínicos.

2.4. Anticoagulantes e Antiplaquetários em Síndrome Coronariana Aguda sem Fibrilação $\underline{\text { Atrial }}$

O estudo APPRAISE- $2^{19}$ verificou em estudo randomizado, duplo-cego, mediana de 6 dias após o diagnóstico de síndrome coronariana aguda sem planejamento de intervenção percutânea imediata, a estratégia do uso de apixaban $5 \mathrm{mg}$ 2 vezes ao dia versus placebo, ambos os grupos utilizando a terapêutica dupla antiplaquetária habitual de aspirina e um tienopiridino.

$\mathrm{O}$ estudo foi interrompido precocemente pela constatação de incremento substantivo das taxas de sangramento sem que houvesse benefício na redução de eventos isquêmicos.

No estudo ATLAS-ACS ${ }^{20}$, testou-se a adição de rivaroxaban ao tratamento da síndrome coronariana aguda.

Neste estudorandomizado, duplo-cego, 15.526 pacientes, em média 7 dias após estabilização de tratamento de síndrome coronariana aguda, sendo $50 \%$ resultado de IAM com supra de ST, e tratamento de revascularização, com stent em sua maioria $(60 \%)$, rivaroxaban na dose de 2,5 mg 2 vezes ao dia foi comparado a rivaroxaban $5 \mathrm{mg} 2$ vezes ao dia e a placebo, todos em uso de terapêutica antiplaquetária dupla, geralmente incluindo aspirina e clopidogrel. O uso da terapia dupla antiplaquetária durou cerca de 13,3 meses, após o que se seguia ao uso da aspirina. $\mathrm{O}$ uso de rivaroxaban foi em média de 13 meses e o acompanhamento do estudo ocorreu por 24 meses.

Os grupos de rivaroxaban, nas doses de 2,5 $\mathrm{mg} 2$ vezes ao dia e $5 \mathrm{mg} 2$ vezes ao dia, combinados, em relação ao placebo, tiveram significativa redução de eventos isquêmicos (morte cardiovascular, infarto do miocárdio ou AVC), em torno de $15 \%$ (de $8.9 \%$ e $10,7 \%$, respectivamente). A mortalidade cardiovascular foi reduzida com a dose de $2,5 \mathrm{mg}$ em $34 \%$ $(\mathrm{p}=0,002)$.

Houve aumento do sangramento com rivaroxaban em comparação ao placebo de $2,1 \%$ e $0,6 \%$, respectivamente.

É importante frisar que neste estudo a taxa de descontinuação do rivaroxaban $2,5 \mathrm{mg} 2$ vezes foi de $26,9 \%$; $5 \mathrm{mg} 2$ vezes ao dia foi de $29,4 \%$; e do placebo, de $26,4 \%$, o que compromete a firmeza das conclusões finais.

Fica a percepção de que talvez em pacientes com síndrome coronariana aguda de alto risco isquêmico deva considerar-se o uso de rivaroxaban como tratamento adjunto em baixas doses.

2.5. Duração da Terapêutica Antiplaquetária Dupla Após o Uso de Stents

Desde o início da angioplastia, que se tornou clara a necessidade do uso de dupla antiagregação plaquetária. $^{21,22}$

\subsubsection{Uso por 1 Mês}

Após o implante de stents, a terapêutica antiplaquetária dupla deverá ser usada por 
um período mínimo de 1 mês para stents metálicos ou stents farmacológicos especiais, como o Biofreedom ${ }^{\mathrm{TM}}{ }^{23}$ ou o Resolute Onix One ${ }^{\mathrm{TM}}$ - estudo apresentado no último TCT 19, utilizando uma plataforma especial para o uso do zotarolimus.

Estudos recentemente publicados, como o STOPDAPT2, ${ }^{24}$ realizado no Japão, e o SMARTCHOICE ${ }^{25}$ realizado na Coreia do Sul, sugerem que em tempos atuais a estratégia de terapêutica antiplaquetária dupla por 1 mês possa ser seguida com a monoterápica com clopidogrel. Especialistas da área chamam a atenção para o fato de que em ambos os estudos o emprego de recurso de apoio à angioplastia com ultrassonografia intracoronária e outros recursos ópticos e funcionais foram utilizados em $100 \%$ dos casos, o que talvez não encontre paralelismo em quase todo o resto do mundo. Uma outra preocupação é a de deixar pacientes com stent sob uso monoterápico de clopidogrel, quando ainda se tem o temor da possibilidade de resistência ou interação de medicamentos e outras substâncias.

\subsubsection{Uso por 3 Meses}

Ouso por 3 meses para pacientes com os demais stents farmacológicos conforme demonstrado nos estudos RESET $^{26}$ e OPTIMIZE ${ }^{27}$ com zotarolimus, foi realizado com uma plataforma mais antiga e a de segunda geração, segundo o estudo STOPDAPT ${ }^{28}$ com everolimus, em pacientes de menor risco de eventos isquêmicos.

\subsubsection{Uso por 6 Meses (maioria dos casos)}

\subsubsection{Uso por 1 Ano ou Mais}

A terapêutica antiplaquetária dupla, mostrase mais adequada por 1 ano ou mais nos casos de maior risco, tais como: história prévia de trombose por stent sob terapia antiplaquetária adequada, angioplastia de artéria "derradeira", doença coronária multiarterial, principalmente em diabéticos, insuficiência renal crônica $(\mathrm{ClCr}<$ $60 \mathrm{~mL} / \mathrm{min}$ ), pelo menos 3 stents e/ou 3 lesões tratadas, ICP em bifurcações, com pelo menos 2 stents colocados, comprimento total da área coberta de stent $>60 \mathrm{~mm}$, tratamento de oclusão coronária crônica.

O estudo DAPT $^{29}$ demonstrou esta adequação quando 9.961 pacientes, após uso de aspirina e clopidogrel ou prasugrel, tiveram o tienopiridínico randomizado comparativamente a placebo após 12 meses de tratamento e até 30 meses, o que mostrou redução de eventos isquêmicos de $5,9 \%$ para $4,3 \%$, com redução de risco relativo de $39 \%(p<0,001)$. Houve redução de todos os componentes dos desfechos primários incluídos como infarto, trombose intra-stent $\mathrm{e}$ mortalidade. Houve considerável aumento, no entanto, de sangramento no grupo que manteve o tienopiridínico de $1,6 \%$ para 2,5\% $(\mathrm{p}<0,001)$.

Este estudo foi publicado em 2014 e houve a utilização de todos os tipos de stents existentes à época, com predomínio do everolimus.

A decisão de utilização de terapêutica prolongada acima de 12 meses leva em conta o risco de isquemia e em contrapartida o risco de sangramento. Escores têm sido criados para ajudar na estimativa de risco de isquemia e de sangramento. $\mathrm{O}$ escore DAPT prevê isquemia e determina como variáveis e suas respectivas pontuações afetam o tratamento: idade $>75$ anos $=-2$ pontos; idade entre 65 e 75 anos $=-1$ ponto; idade $<65$ anos $=0$; tabagismo $=1$; diabetes mellitus=1; IAM na apresentação inicial=1; IAM ou IPC prévia $=1$; diâmetro do stent $<3 \mathrm{~mm}=1$; stent com placlitaxel $=1$; insuficiência cardíaca ou FE reduzida $=2$ e enxerto de ponte de safena $=2$.

Escore $\geq 2$ favorece o emprego de terapia antiplaquetária dupla por 30 meses.

Por outro lado, o escore PRECISE DAPT ${ }^{30}$ foi criado utilizando 5 indicadores como taxa de hemoglobina, idade, contagem de leucócitos, clearance de creatinina e história de sangramento prévio, permitindo a determinação de um escore que, quando acima de 25, recomenda um uso mais curto de terapia dupla, de 3 a 6 meses, e quando menor com risco isquêmico alto, de 12 a 24 meses. Estes escores podem ser encontrados 
como aplicativos: DAPT Risk Calculator e PRECISEDAPT.com.

Osnovosantiplaquetários apresentamum custo mais elevado que o clopidogrel. O estudo recente, aberto e unicêntrico TOPIC (Timing of Platelet Inhibition After Acute Coronary Syndrome), despertou algum interesse nesse sentido, em que 646 pacientes foram randomizados pós síndrome coronariana aguda, tratada com stents, para uma estratégia de terapia antiplaquetária com aspirina e um dos novos antiplaquetários, ticagrelor ou prasugrel, por 1 ano, ou após 1 mês de terapêutica dupla com aspirina e ticagrelor ou prasugrel, sem sangramento; e tiveram o novo antiplaquetário substituído por clopidogrel, sendo igualmente acompanhados por 1 ano.

Os resultados do estudo demonstraram que o máximo do benefício dos novos antiplaquetários com ação anti-isquêmica mais potente que o clopidogrel se dá no primeiro mês de tratamento e que a partir daí não demonstram maior eficácia anti-isquêmica e até aumentam o risco de sangramento.

O desfecho primário do estudo foi uma combinação de morte cardiovascular, cirurgia de revascularização miocárdica urgente, AVC, ou sangramento BARC maior que $2 \mathrm{em} 1$ ano de acompanhamento e ocorreu em $13,4 \%$ dos pacientes que fizeram a troca, e $26,3 \%$ para os que mantiveram o mesmo esquema inicial $(\mathrm{p}<0,01)$. Em uma análise individualizada de desfechos, verifica-se que a diferença se deveu à incidência de sangramento que foi maior no grupo da terapêutica com novo antiplaquetário e aspirina do que no grupo que mudou para aspirina e clopidogrel depois de 1 mês de tratamento $(23,5 \%$ versus $9,3 \%$, respectivamente, $p<0,01)$.

Embora este estudo apresente as restrições por se tratar de um estudo aberto e unicêntrico, ainda assim pode trazer alento para o atingimento de beneficio máximo do tratamento antiplaquetário duplo em que se faça necessário o seu uso prolongado em pacientes com alguma dificuldade financeira para aquisição do novo antiplaquetário mais caro, lembrando que foi 1 mês após uso do esquema de aspirina e um novo antiplaquetário por 1 mês em pacientes que neste período não apresentaram sangramento.

\subsection{Antiplaquetários Após Uso de Stents em} Procedimentos Eletivos

As evidências positivas dos estudos de síndrome coronariana aguda mostrando superioridade do ticagrelor e prasugrel sobre o clopidogrel tem levado a uma utilização off-label desses produtos na condição de doença estável, sem que tenha sido construída uma evidência científica para tal, exceto em subgrupos de estudos. O estudo ALPHEUS (Assessment of Loading with the P2Y12 Inhibitor Ticagrelor or Clopidogrel to HaltI Schemic Events in Patients Undergoing Elective Coronary Stenting) irá prover informações mais definitivas a esse respeito.

Em pacientes de maior risco de sangramento, o uso de terapia antiplaquetária dupla é preocupante por conta do aumento de hemorragias associadas ao seu emprego, com consequências reconhecidamente importantes pela hemorragia em si, assim como por sangramento intracraniano.

Em pacientes com alto risco isquêmico e também alto risco de sangramento, seria desejável ter preservada a ação anti-isquêmica sem aumento do risco de sangramento. A possibilidade de uso de um novo antiplaquetário como monoterapia, sem aspirina associada talvez possa cumprir esse papel em tais pacientes.

O estudo recentemente publicado, TWILIGHT, ${ }^{31}$ testou essa hipótese em 7.119 pacientes considerados de maior risco para isquemia e sangramento após intervenção percutânea: cerca de $65 \%$ com IAM sem supra ou angina instável apresentarem ao menos um dos fatores adicionais de risco (por exemplo: idade $>65$ anos, sexo feminino, tipos de lesões coronarianas, presença de disfunção renal ou $\mathrm{DM})$ e que foram tratados por 3 meses com ticagrelor e aspirina revestida. Foram então excluídos os pacientes que tivessem apresentado 
neste período um sangramento significativo. Os demais pacientes foram randomizados para ticagrelor e aspirina ou ticagrelor apenas por um período de 12 meses adicionais.

Os resultados foram de redução do desfecho de sangramento de $44 \%$ de $7,1 \%$ para $4 \%$ (p < 0,001), considerando-se os critérios BARC 2,3 ou 5. Convém registrar que o mesmo ocorreu com todos os mesmos critérios de sangramento utilizados no estudo e com ocorrência de evento isquêmico de 3,9\% para ambos os grupos.

\subsection{O Uso de Antiplaquetários no Portador de DAC com Fibrilação Atrial}

Sabe-se que na fibrilação atrial os anticoagulantes são superiores aos antiplaquetários na prevenção de embolismo sistêmico. Os anticoagulantes varfarínicos podem substituir a aspirina em casos de DAC crônica, prevenindo a um só tempo eventos sistêmicos embólicos e eventos coronarianos. ${ }^{32}$ Com o surgimento dos novos anticoagulantes espera-se reproduzir os mesmos achados.

Por outro lado, em situações de muito alto risco trombótico coronariano, como na síndrome coronária aguda e em colocação de stents, verificou-se há muito que se faz necessário o uso de terapêutica anticoagulante com heparina e dupla antiagregação plaquetária, o que previsivelmente é acompanhado de aumento de risco de sangramento.

$\mathrm{O}$ interesse tem sido em verificar nessas condições por quanto tempo deverá permanecer a terapêutica tríplice, anticoagulante e dois antiplaquetários, e em seguida qual o melhor esquema de antiplaquetário se agrega ao anticoagulante, em terapêutica combinada simples e, posteriormente, qual o anticoagulante que permanecerá sozinho: o antivitamina $\mathrm{K}$ ou um dos novos anticoagulantes.

NoestudoPIONEERAF-PCI, ${ }^{33} 2.124$ pacientes com FA em menos de $72 \mathrm{~h}$ após realização de angioplastia para SCA foram randomizados em 3 grupos (aproximadamente metade dos casos): rivaroxaban $15 \mathrm{mg} 1 \mathrm{vez}$ ao dia e um inibidor do
P12Y12, ou rivaroxaban 2,5 $\mathrm{mg} 2$ vezes ao dia e aspirina e um inibidor do P2Y12 ou varfarina, além de terapêutica antiplaquetária dupla. O inibidor de P2Y12 foi clopidogrel em quase a totalidade dos casos.

Os grupos do rivaroxaban apresentaram menos sangramento ao final do acompanhamento e, embora a eficácia anti-isquêmica parecesse semelhante entre os 3 grupos testados, o intervalo de confiança nos resultados foi largo o bastante para não garantir com certeza que não pudesse haver diferença entre eles, sendo, portanto, o estudo inconclusivo em relação a este aspecto.

No estudo RE-DUAL, ${ }^{34}$ logo após tratamento por angioplastia (eletiva em $44,1 \%$ dos casos), os pacientes foram randomizados para 3 regimes possíveis: 2 grupos com dabigatran $150 \mathrm{mg}$ ou $110 \mathrm{mg} 2$ vezes ao dia (todos os pacientes nos EUA foram randomizados para as 2 doses, exceto os mais idosos com $>80$ anos e população no Japão) e uso de clopidogrel ou ticagrelor; e 1 grupo com varfarina em terapia tripla com aspirina e clopidogrel ou ticagrelor.

Assim, os grupos do dabigatran sempre tiveram terapia dupla, com 110 ou $150 \mathrm{mg} 2$ vezes ao dia e sem aspirina enquanto que o grupo da varfarina fez terapêutica tripla por 1 mês em caso de stents metálicos ou 3 meses em caso de stents farmacológicos quando a aspirina foi suspensa, permanecendo a varfarina com clopidogrel ou ticagrelor.

O desfecho primário do estudo foi taxa de sangramento de $15,4 \%$ em terapia dupla com dabigatran $110 \mathrm{mg}$ e $20,2 \%$ no grupo de dabigatran $150 \mathrm{mg}$; e, em torno de $26 \%$, no grupo da terapêutica tríplice que incluiu a varfarina, aspirina e clopidogrel ou ticagrelor com significante diferença estatística entre os grupos do dabigatran e o grupo da varfarina.

$\mathrm{O}$ evento isquêmico aparentemente foi semelhante nos 3 grupos.

Como a taxa de sangramento foi maior no grupo da varfarina logo nos primeiros meses de observação quando havia o uso de varfarina e aspirina, persite a dúvida se esta se deveu ao 
emprego da varfarina ou da aspirina.

Mais recentemente, o estudo ENTRUST-AF $\mathrm{PCI}^{35}$ randomizou 1.506 pacientes com FA e angioplastia coronariana $45 \mathrm{~h}$ após intervenção percutânea: $52 \%$ com síndrome coronariana aguda, 4 a 5 dias após a angioplastia, para o uso de endoxaban $60 \mathrm{mg}$, ou $30 \mathrm{mg}$, para os pacientes com clearance reduzido de 15 a $50 \mathrm{~mL} / \mathrm{min}$ ou peso menor que $60 \mathrm{~kg}$, e um inibidor do P2Y12, predominantemente o clopidogrel, por 12 meses, em comparação ao uso de anticoagulante antivitamina $\mathrm{K}$ e aspirina e inibidor do P2Y12, geralmente o clopidogrel, por um período variável de 1 a 12 meses de acordo com o tipo do stent.

O desfecho primário foi sangramento maior ou menor relevante e uma taxa anualizada de $20,7 \%$ com endoxaban e $25,6 \%$ com o outro regime, com redução numérica de $17 \%$ de risco relativo que não atingiu significância estatística para superioridade $(p=0,11)$, porém demonstrou não-inferioridade. $\mathrm{O}$ desfecho isquêmico foi aparentemente semelhante entre os dois grupos.

O estudo AUGUSTUS ${ }^{36}$ ajudou a consolidar a informação do emprego de antitrombóticos no contexto de FA e DAC aguda ou de IPC. Seu delineamento foi mais bem feito, pois tratou de maneira bifatorial a comparação de apixaban versus varfarina, e aspirina versus placebo. Assim, dos 4.614 pacientes com FA e síndrome coronariana aguda, $\sim 60 \%$ ocorreu em pacientes tratados ou não com IPC, e $40 \%$ com IPC eletiva.

Os pacientes foram randomizados em forma aberta para apixaban $5 \mathrm{mg} 2$ vezes ao dia ou 2,5 2 vezes ao dia se tivesse 2 de três critérios prédefinidos: idade $>80$ anos, peso menor que 60 $\mathrm{kg}$ ou creatinina $>1,5 \mathrm{mg}$ ou para varfarina. $\mathrm{O}$ inibidor do P2Y12, geralmente o clopidogrel, esteve em uso durante todo o período do estudo de 6 meses.

O resultado mostrou que o desfecho primário de sangramento maior ou relevante como definido pelo International Society on Thrombosis and Haemostasis (ISTH) favoreceu o apixaban em comparação à varfarina, com redução de $31 \%$ de eventos $(\mathrm{p}<0,001)$ e foi maior em $89 \%$ com a aspirina em relação ao placebo. Não houve diferença de eficácia entre os grupos.

\subsection{Terapêutica Antitrombótica em FA e DAC Estável}

O estudo OAC-ALONE ${ }^{37}$ foi planejado para incluir 2.000 pacientes em 12 meses, testando varfarina ou 1 dos 4 novos anticoagulantes associados à aspirina ou ao clopidogrel em paciente com FA e DAC estável. O estudo foi inconclusivo, pois foi interrompido precocemente com a admissão de apenas 696 pacientes, dada a dificuldade de inclusão de pacientes em 38 meses.

O estudo AFIRE, ${ }^{38}$ recentemente publicado, é mais definitivo em relação ao tema. Realizado no Japão, o estudo, com 2.236 pacientes portadores de FA e que tivessem se submetido a intervenção percutânea ou cirurgia de revascularização miocárdica há mais de 1 ano, ou que tivessem documentação angiográfica de DAC permanecendo em tratamento clínico em quadro estável, testou o uso comparativo de monoterapia com rivaroxaban $15 \mathrm{mg} /$ dia ou 10 mg para pacientes com clearance de creatinina entre 15 e $49 \mathrm{mg} / \mathrm{min}$ ou sua combinação com aspirina $(70,2 \%)$ ou um inibidor do P2Y12 $(26,2 \%)$ à discrição do médico assistente, por um período de até 36 meses. Os resultados foram melhores para a monoterapia com anticoagulante tanto para os desfechos primários de eficácia, considerados morte por qualquer causa ou eventos cardiovasculares, que ocorreram em $4,14 \%$ e $5,75 \%$ para monoterapia versus terapia combinada $(\mathrm{p}<0,001)$, assim como para o efeito primário de segurança sob a forma de sangramento de $1,62 \%$ e $2,76 \%$ para monoterapia versus terapia combinada $(\mathrm{p}<0,01)$. Este estudo foi interrompido precocemente dada a superioridade do tratamento monoterápico com anticoagulante (rivaroxaban) combinado a um antiplaquetário.

Este estudo reforça a indicação com base em um estudo randomizado, o que já vinha sendo 
feito até então em diretrizes com base em estudos observacionais ou de pequeno porte.

\section{Suspensão de Antiplaquetário para Procedimentos}

A suspensão de antiplaquetários face a necessidade de procedimentos cirúrgicos eletivos deve levar em conta o balanço entre o risco isquêmico determinado por sua suspensão e o benefício de diminuir a chance de hemorragia mais severa.

Não existem estudos conclusivos sobre o assunto. ${ }^{39}$

Em cirurgias eletivas, pensa-se em suspender o antiplaquetário em cirurgias de maior porte, como as torácicas e abdominais, ou de locais de risco mais delicado, como sistema nervoso central ou de superfícies não-compreensíveis. A aspirina nessas condições deverá ser suspensa 5 a 7 dias antes do procedimento; o clopidogrel por 7 dias e o ticagrelor e prasugrel por 3 a 5 dias.

\section{Referências}

1. Kurlansky PHM, Prince S, Mack M. Coronary artery bypass graft versus percutaneous coronary intervention meds matter: impact of adherence to medical therapy on comparative outcomes. Circulation. 2016;134:12381246.

2. Boden WE ORR, Teo KK, et al. Optimal medical therapy with or without PCI for stable coronary disease. N Engl J Med. 2007;356:1503-1516.

3. Jr V. Inhibition of prostaglandin synthesis as a mechanism of action of aspirin like drugs. Nat New Biol. 1971;231:232-5.

4. CLASSICS Investigators BME, Rupprecht HJ, Urban P, Gershlick AH. Double-blind study of the safety of clopidogrel with and without a loading dose in combination with aspirin compared with ticlopidine in combination with aspirin after coronary stenting: the CLopidogrel ASpirin Stent International Cooperative Study (CLASSICS). Circulation. 2000:624-629.

5. Collaboration AT. Antithrombotic Trialists' Collaboration Collaborative. Meta-analysis of randomised trials of antiplatelet therapy for prevention of death, myocardial infarction, and stroke in high risk patients. BMJ 2002;324:71-86.
6. Committee CS. A randomised, blinded, trial of clopidogrel versus aspirin in patients at risk of ischaemic events (CAPRIE). Lancet. 1996;348:13291339.

7. Bhatt FK, Hacke W, Berger PB, Black HR, Boden WE et al. CHARISMA Investigators. Clopidogrel and aspirin versus aspirin alone for the prevention of atherothrombotic events. NEngl J Med. 2006;354:170617.

8. Bonaca BD, Cohen $\mathrm{M}$ et al. PEGASUS-TIMI 54 Steering Committee and Investigators. Long-term use of ticagrelor in patients with prior myocardial infarction. N Engl J Med. 2015;372:1791-1800.

9. Group I-SISoISC. Randomised trial of intravenous streptokinase, oral aspirin, both, or neither among 17,187 cases of suspected acute myocardial infarction: ISIS-2. Lancet. 1988;ii:349-360.

10. Yusuf SZF, Mehta SR, Chrolavicius S, Tognoni G, Fox KK. Effects of clopidogrel in addition to aspirin in patients with acute coronary syndromes without ST-segment elevation. N Engl J Med. 2001;345:494502.

11. Group CC. Addition of clopidogrel to aspirin in 45852 patients with acute myocardial infarction: randomised placebo-controlled trial. Lancet. 2005;366:1607-21.

12. Sabatine MS CC, Gibson CM, López-Sendón JL, Montalescot G, Theroux P et al. CLARITY-TIMI 28 Investigators. Addition of clopidogrel to aspirin and fibrinolytic therapy for myocardial infarction with ST-segment elevation. N Engl J Med. 2005;352:1179118.

13. Investigators TC-O. Dose comparisons of clopidogrel and aspirin in acute coronary syndromes. N Engl $\mathrm{J}$ Med. 2010;363:930-942.

14. Wallentin L BR, Budaj A, Cannon CP, Emanuelsson H, Held C, et al. PLATO Investigators. Ticagrelor versus clopidogrel in patients with acute coronary syndromes. N Engl J Med. 2009;361:1045-57.

15. Montalescot GWS, Braunwald E, Murphy SA, Gibson $\mathrm{CM}, \mathrm{McCabe} \mathrm{CH}$ et al. TRITON-TIMI 38 investigators. Prasugrel compared with clopidogrel in patients undergoing percutaneous coronary intervention for ST-elevation myocardial infarction (TRITON-TIMI 38): double-blind, randomised controlled trial. Lancet. 2009;373:723-31.

16. Montalescot GBL, Dudek D, Goldstein P, Hamm C, Tanguay JF etal. ACCOAST Investigators. Pretreatment with prasugrel in non-ST-segment elevation acute coronary syndromes. N Engl J Med. 2013;369:9991010.

17. Roe MT AP, Fox KA, et al., on behalf of the TRILOGY ACS Investigators. Prasugrel versus clopidogrel for acute coronary syndromes without revascularization. N Engl J Med. 2012;367:1297-1309. 
18. Schüpke SNF-J, Menichelli M et al. Ticagrelor or prasugrel in patients with acute coronary syndromes. N Engl J Med. 2019;381:1524-1534.

19. Alexander LRD, James $\mathrm{S}$ et al. Apixaban with antiplatelet therapy after acute coronary syndrome. N Engl J Med. 2011;365:699-708.

20. Mega BE, Mohanavelu S et al. Rivaroxaban versus placebo in patients with acute coronary syndromes (ATLAS ACS-TIMI 46): a randomised, double-blind, phase II trial. Lancet. 2009;374:29-38.

21. Schomig ANF, Kastrati A, Schuhlen H, Blasini R, Hadamitzky M, Walter H, Zitzmann-Roth EM, Richardt G, Alt E, Schmitt C, Ulm K. A randomized comparison of antiplatelet and anticoagulant therapy after the placement of coronary-artery stents. New Engl J Med. 1996;334:1084-1089.

22. Leon MBBD, Popma JJ, Gordon PC, Cutlip DE, Ho KK et al. A clinical trial comparing three antithromboticdrug regimens after coronary-artery stenting. Stent Anticoagulation Restenosis Study Investigators. N Engl J Med. 1998;339:1665-1671.

23. Urban PMI, Abizaid A et al. Polymer-free drug-coated coronary stents in patients at high bleeding risk. N Engl J Med. 2015;373:2038-2047.

24. Hirotoshi MTD, Takeshi M, Masahiro N, Hiroki S. Toshiaki $\mathrm{T}$ et al. Effect of 1-month dual antiplatelet therapy followed by clopidogrel versus 12-month dual antiplatelet therapy on cardiovascular and bleeding events in patients receiving PCI. The STOPDAPT-2 Randomized Clinical Trial. JAMA 2019;321:24142427.

25. Joo-Yong Hahn MYBS, Ju-Hyeon Oh, Woo Jung Chun, Yong Hawn Park, Woo Jin Jang. Effect of P2Y12 inhibitor monotherapy versus dual antiplatelet therapy on cardiovascular events in patients undergoing percutaneous coronary intervention. The SMART-CHOICE Randomized Clinical Trial. JAMA 2019;321(24):. 2019;321:2428-2437.

26. Kim BKHM, Shin DH, Nam CM, Kim JS, Ko YG et al. A new strategy for discontinuation of dual antiplatelet therapy: the RESET Trial (REal Safety and Efficacy of 3-month dual antiplatelet Therapy following Endeavor zotarolimus-eluting stent implantation). J Am Coll Cardiol 2012;60:1340-1348.

27. Feres FCR, Abizaid A, Leon MB, Marin-Neto JA, Botelho RV et al. Three vs twelve months of dual antiplatelet therapy after zotarolimus-eluting stents: the OPTIMIZE randomized trial. JAMA. 2013;310:25102522.

28. Natsuaki MT, Yamamoto E, Shiomi H, Furukawa $\mathrm{Y}$, Abe $\mathrm{M}$ et al. One-year outcome of a prospective trial stopping dual antiplatelet therapy at 3 months after everolimus-eluting cobalt-chromium stent implantation: short and optimal duration of dual antiplatelet therapy after everolimus-eluting cobaltchromium stent (STOPDAPT) trial. Cardiovasc Interv Ther. 2016;31:196-209.

29. Mauri LKD, Yeh RW, Driscoll-Shempp P, Cutlip DE, Steg PG et al. Twelve or 30 months of dual antiplatelet therapy after drug-eluting stents. $\mathrm{N}$ Engl J Med. 2014;371:2155-2166.

30. Costa FKD, James $\mathrm{S}$ et al. Derivation and validation of the predicting bleeding complications in patients undergoing stent implantation and subsequent dual antiplatelet therapy (PRECISE-DAPT) score: a pooled analysis of individual-patient datasets from clinical trials. Lancet. 2017;389:1025-1034.

31. Mehran RBU, Sharma SK et al. Ticagrelor with or without aspirin in high-risk patients after PCI. N Engl J Med. 2019;381:2032-42.

32. Anand SSYS. Oral anticoagulants in patients with coronary artery disease. J Am Coll Cardiol. 2003;41:62S-69S.

33. Gibson CMMR, Bode $\mathrm{C}$ et al. Prevention of bleeding in patients with atrial fibrillation undergoing PCI. N Engl J Med. 2016;375:2423-2434.

34. Cannon CPBD, Oldgren J et al. Dual antithrombotic therapy with dabigatran after PCI in atrial fibrillation. N Engl J Med. 2017;377:1513-1524.

35. Vranckx PVM, Eckardt L, Tijssen J, Lewalter T, Gargiulo $G$ et al. Edoxaban-based versus vitamin $\mathrm{K}$ antagonist-based antithrombotic regimen after successful coronary stenting in patients with atrial fibrillation (ENTRUST-AF PCI): a randomised, open-label, phase 3b trial. Lancet. 2019;394:13351343.

36. Lopes RD HG, Aronson R, Vora AN, Massaro T, Mehran R et al. Antithrombotic therapy after acute coronary syndrome or PCI in atrial fibrillation. N Engl J Med. 2019;380:1509-1524.

37. Matsumura-Nakano YSS, Komasa A et al. Open-label randomized trial comparing oral anticoagulation with and without single anti- platelet therapy in patients with atrial fibrillation and stable coronary artery disease beyond 1 year after coronary stent implantation. Circulation. 2019;139:604-16.

38. Yasuda SKK, Akao M et al. Antithrombotic therapy for atrial fibrillation with stable coronary disease. N Engl J Med. 2019;381:1103-13.

39. Childers CP, Maggard-Gibbons M, Ulloa JG et al. Perioperative management of antiplatelet therapy in patients undergoing non-cardiac surgery following coronary stent placement: a systematic review. Systematic Reviews. 2018;7:1-12. 